\title{
Male-Female Wage Gap and Vertical Occupational Segmentation: the Role of Work Attitude
}

\author{
Olivier Baguelin *
}

In this paper, we provide a theoretical model of employment relation to capture the role of work attitudes in male-female disparities in the labour market. Our point is that this aspect, although often overlooked in the economic literature, may be crucial to understand male-female occupational and wage differences. According to an OECD study (2002, p.65), on average over thirteen European countries, once the effects of education, tenure, potential experience and other observable characteristics are controlled for, gross hourly wages are still 16\% higher for men than for women. This gap can be imputed to two sources: unobserved differences in gender characteristics and/or wage discrimination against women. Yet, the assumption of a pure pay discrimination against women (i.e. of a systematically lower female wage for a given job within a given company) is generally invalidated by empirical studies ${ }^{1}$. An explanation of male-female disparities in the labour market consistent with the data, thus, should go through unobserved differences in gender characteristics. Male-female segmentation of the labour market being widely documented, notably in its vertical dimension ${ }^{2}$, authors suspect differences between the jobs mostly held by men or by women to represent a significant source of gender differences in unobserved characteristics. This suspicion is strengthened by the observation of a strong and significant correlation between the unexplained part of the gender wage gap and a dissimilarity index for each country ${ }^{3}$ : the more dissimilar male and female workforces occupational distribution, the wider average wage gap. Occupational segmentation then seems crucial to understand male-female disparities in the labour market ${ }^{4}$. In a similar spirit, Macpherson and Hirsch (1995) document the "gender composition effect"

Centre d'études de l'emploi March 9, 2010

See, for the United-States, Johnson and Solon (1986) or Macpherson and Hirsch (1995)

See OECD (2002, pp. 85-95)

3 Measuring the extent to which the labour force can be divided into gender-dominated and gender-integrated occupations.

4 See OECD (2002, pp. 85-95). This conclusion meets that of Johnson and Solon (1986) who show, for the United-States, that gender wage differences result more from the fact that men are hired in firms which pay well compared to those where women predominate than from pure pay discrimination within given firms. See Blau and Kahn (2002) for more references. 
showing that wage levels are substantially lower in predominantly female occupations. The longitudinal analysis they conduct, indicates that twothird or more of the standard gender composition effect is accounted for by occupational characteristics and unmeasured worker skill or taste differences. The issue is therefore to understand "how and why the labor market sorts women and men into jobs with different characteristics and productivity" ${ }^{5}$. The present work aims to propose an interpretation of male-female disparities meeting this concern. More precisely, we provide a micro model of how occupational characteristics and unmeasured workers differences combine in the emergence of gender vertical occupational segmentation.

Among the unobserved individual characteristics that could make a difference between male and female workers in the labour market, the OECD study mentioned above points out motivation (OECD, p. 101): men and women possibly develop a different motivation to work and effort. Motivation indeed stands as a major unobserved productivity-relevant characteristics (as such, its effects are reflected in the residual of the wage gap decomposition). Furthermore, as required by Macpherson and Hirsch's results, work motivation is at the intersection of skill and taste, and probably correlated with occupational characteristics. The assumption of malefemale differences in work attitude, thus, seems worth studying. And yet, it has received little attention in conventional models. The fact is that, within this perspective, motivation and incentives are inseparable: a malefemale difference in motivation can only result from... a difference in wage incentives, which is precisely what needs to be explained. The OECD report obviously rather refers to non pecuniary forms of work motivation, which cannot be captured with usual assumptions. The model introduced below is meant to capture such a non pecuniary work motivation. Many research avenues have been opened on this line, notably among the proponents of the behavioral approach in economics. Fehr and Falk (2002) provide a variety of experimental data putting forward the relevance, within the framework of an employment relation of: such motives as reciprocity, the need for social approval, the observing of social norms; phenomena such as intrinsic motivation. Frey (1997) analytically studies the economic issues of intrinsic motivation and its sensitivity to incentives. Within a cognitive perspective, Bénabou and Tirole (2006) study the link between self-esteem and motivation through a signalling and self-signalling argument: some actions of agents are aimed at signalling and/or self-signalling an ideal self-image, valued in itself in terms of well-being. This latter work follows the inspiration of the more applied analyses of Akerlof and Kranton (2000, 2005) who directly rely on the conclusions of social psychology using the notion of identity as a tool for economic analysis.

5 Macpherson and Hirsch (1995, p. 463). 
In the present paper, we develop a simple principal-agent model intended to conveniently capture the notion of a non pecuniary work motivation ${ }^{6}$. This is done within a "reduced form perspective", close to that of Akerlof and Kranton (2000, 2005) who consider agents with identity and self-esteem motives. In our model, the agent (hereafter, he) faces with a (possibly unconscious) choice binding his self-esteem motives. In psychological terms, this corresponds to the choice of a context of self-evaluation, ${ }^{7}$ that is, of a social context relative to which the agent builds his conception of his self-esteem. We reduce this choice to that of an identity more or less polarized towards working life. When this polarity is strong, self-esteem motives adds to usual ones and the agent exhibits a non pecuniary work motivation. Although the principal (hereafter, she) may take advantage of this extra work motivation, she is constrained by the characteristics of the job to be filled. We focus on two characteristics, productivity and working conditions, which impact wage but are generally not precisely observed empirically (and so, often confined in the residual of wage regressions). We study the impact of these characteristics on the human ressources management strategy of the principal (inducing a non pecuniary work motivation or not).

Equipped with such a model, it becomes possible to study virtual differences in work motivation between male and female workers. We can then address the issue of male-female disparities in the labour market in a way consistent with empirical knowledge. Our starting assumption is that women have more opportunity than male to enjoy self-esteem outside work, all other things being equal. A direct consequence is that employment terms disregarding non pecuniary work motivation can only be accepted by female workers, not by males. This assumption then, leads to a gender segmented occupational distribution with male workers confined to jobs inducing a non pecuniary work motivation. When jobs vary depending on their productivity, this involves an overrepresentation of female workers in the least productive jobs. When jobs vary depending on working conditions, this involves an overrepresentation of female workers in the least effort-intensive jobs. In both cases, female workers are overrepresented in poorly paid jobs which induces an average earnings gap in favor of men.

François (1998) also provides a theoretical analysis of male-female disparities in the labour market rooted in gender occupational segmentation. He does it within a conventional perspective (purely pecuniary motivation) from an efficiency wage model à la Shapiro-Stiglitz with (beckerian) domestic production. Within this framework, the labour market may be

6 By "non pecuniary work motivation", we refer to a work motivation not related to any consumption plan. Although, the notion includes intrinsic motivation, in the remaining, we are mostly interested in a work motivation resulting from factors such as occupational prestige or social status which can impact self-esteem. This allows to capture a non standard impact of wage and effort on behaviour.

7 See Gecas and Seff (1990). 
gender segmented, male workers being overrepresented in jobs paying an efficiency wage. François is particularly interested in the impact of marital status on wage, and his model does well in capturing the empirical finding of a wage premium for married men but not for married women. By inserting non-pecuniary work motivation in the analysis, taking attitudes into account offers a complementary more individualistic analysis.

More generally, the beharioral approach does a good job in capturing discontinuities in behaviors. In this respect, a crucial insight is that satisfaction is referential i.e. that individuals evaluate their lot by considering some reference (the average lot in a neighbourhood, a social norm, a past situation, etc.). Within the employment relation framework, workers' satisfaction derives from the comparison of their actual wage and effort $(w, e)$ to some reference $(\tilde{w}, \tilde{e})$, and their behaviour (responses to incentives) depends on the relative position of $(w, e)$ and $(\tilde{w}, \tilde{e})$. This raises the problem of choosing $(\tilde{w}, \tilde{e})$. Considering an employment relation, Akerlof and Kranton (2005) introduce a reduced form model of self-esteem with exogenous $\tilde{e}$. Bénabou and Tirole (2006) rely on a psychology-inspired signaling game in which the self-esteem experienced by a bayesian agent derives from the belief about his type ("shirker" or "conscientious") as resulting from $e$ : when a separating equilibrium exists, $\tilde{e}$ represents the endogenous effort threshold over which the agent enjoys a positive self-image. The model has only one decision-maker and is not so tractable when considering an interaction. Our analysis is somewhere else between the two latter studies: it provides a simple reduced form model of employment relation with $(\tilde{w}, \tilde{e})$ sensitive to the occupational context of the interaction.

Our study decomposes into four steps. In the first, we provide some empirical micro findings about male-female differences in (subjective and objective) working experience. The second step is devoted to presenting the basic model. In the third step, we conduct the analysis of the employment relation with virtual non pecuniary work motivation. The fourth section addresses the issue of male-female occupational and earnings disparities.

\section{Some empirical micro findings about male-female differences in working experience}

Gecas and Seff (1990) show that home and family are two meaningful contexts of self-evaluation. They find that when work was a central aspect of men's self-concept, occupational variables (occupational prestige, control at work) were more strongly related to self-esteem than when they were not; similarly, when home was important, home variables (control and satisfaction at home) were strongly related to self-esteem. Using U.S. longitudinal data, Hakim (2000) studies the long term impact of aspirations and life 
goals as measured by preferences for a life centered on marriage and children or an employment career. It is shown that, twenty years after expressing them, most women had achieved their objectives, resulting in a dramatic advantage of career planners in terms of occupational grade and earnings. Furthermore, female career planners were more likely to choose typically male jobs, and had lower satisfaction than other women (like men, generally). Hakim's point is that, in modern societies, the contraceptive revolution of the 60's has allowed women to take control of their fertility, and gave access to a geniune choice as regards their lifestyle. Hakim (2000) shows that, at all levels of education and in all social classes, women choose among three different lifestyles: adaptative, work-centered, or home-centered. Adaptative women prefer to combine employment and family work without giving a fixed priority to either; they are generally the largest group. Work-centered people (men or women) are focused on competitive activities in the public sphere; the majority of men are work-centered, compared to a minority of women. Home-centered women prefer to give priority to private family life; this group is a minority.

Lambert (1991) exploits a U.S. survey devoted to male-female differences as regards subjective experience of work. The main issue is to understand why men and women maintain comparable levels of job satisfaction even though women's jobs are less gratifying in terms of both intrinsic and extrinsic rewards ${ }^{8}$. A first aspect of Lambert's contribution is to confirm previous findings: women appear to place a higher value than men on social relationships in the workplace, while men place greater importance on career-related job features such as pay, advancement, and autonomy. Lambert finds that by taking into account the opportunities for social satisfaction provided by ones job and the stress resulting from given employment terms, male workers' jobs are not more rewarding than those of female. Controlling for the impact of employment terms, men and women have similar levels of work satisfaction. If the jobs held by women are, in the average, less pecuniary rewarding, they are also less stressful and provide more social satisfaction. As Lambert (1991, p.360) observes "Men are more likely to be both inundated with, and more sensitive to, conflicting and overwhelming job tasks, resulting in greater psychological involvement in work at the cost of reduced job satisfaction."

Sociological literature ${ }^{9}$ suggests that the boundaries between work and family are asymmetrically permeable for men and women. It is argued that men allow the demands of work to intrude more into family life than vice versa, whereas women permit the demands of family life to intrude

8 See Hakim (2003, p. 370): "In all modern societies, studies invariably show higher levels of job satisfaction among women than among men, even when women are in jobs that have lower status and lower pay than men's jobs."

9 See the short survey in Lamber (1991). 
more into work life. Lambert's (1991, p. 360) interpretation of gender diverging subjective work experience is that: "Men may expect jobs to help them perform their breadwinning roles, while women may expect jobs not to interfere with their caregiving roles. Consequently, men and women may place higher value on those job characteristics which help them fulfil these different roles, leading them to maintain employment in jobs with very different characteristics."

The OECD (2002, p. 93) report mentioned in introduction summons up studies suggesting an under-utilization of women skills at work. In spite of educational attainment levels that are similar for women and men or even in favour of women, white-collar women engage in writing and reading at work less frequently and/or with less variety than white-collar men in all OECD countries examined; fewer women than men declare that they are carrying out complex tasks in their jobs; more women than men feel that the demands imposed on them by their jobs are too low relative to their skills, conversely, fewer women than men think they are too high. Overall, it seems that men's jobs are in the average more effort-intensive than women's.

\section{The basic model}

The basic model is designed so as to meet two main criteria: remaining as close as possible to the most standard model of employment relation; conveniently capturing the notion of a non pecuniary work motivation.

\subsection{Basic assumptions}

There are two players: a principal (the employer, she) and an agent (the employee, $h e$ ). When she transfers $w$, the agent exerting effort $e$, her net surplus is given by $S(e)-w$ where $S($.$) denotes the gross surplus function$ associated to the job. We assume that effort is verifiable and that $e$ and $w$ cannot be negative (the agent's liability is limited). The agent, consciously or not, chooses the relation he has to his job. This is modeled as the choice of an identity. There exist two polar identities $A$ and $B$; the agent can make any combination of these two. $a(w, e)$ is the self-esteem (be it intrinsic or resulting from social esteem) he enjoys, assuming he has identity $A$, exerts effort $e$, and earns $w$. When an agent has identity $B$, his self-esteem is taken exogenous set to $b>0$. The well-being of an agent who has chosen the identity combination $\mathrm{x} \in[0,1]$, exerting effort $e$, and earning $w$ writes:

$$
u^{\mathrm{x}}(w, e)=f\left(w, e, x^{\mathrm{x}}(w, e)\right)
$$


where $x^{\mathrm{x}}(w, e)$ combines the amounts of self-esteem $a(w, e)$ and $b$ such that $x^{1}(w, e)=a(w, e)$ and $x^{0}(w, e)=b$. We assume that $f($.$) and a($. are twice differentiable in each of their arguments and ${ }^{10}: f_{w}^{\prime}>0, f_{e}^{\prime}<0$ and $f_{x}^{\prime}>0 ; a_{w}^{\prime}>0$, and $a_{e}^{\prime}>0$; for all $x \in[0,1], \frac{\partial u^{x}}{\partial e}<0$. It is further assumed that for all $x \in[0,1]: \frac{\partial^{2} u^{x}}{\partial e^{2}} \leq 0, \frac{\partial^{2} u^{\mathrm{x}}}{\partial w^{2}} \leq 0$ and $\frac{\partial^{2} u^{\mathrm{x}}}{\partial e \partial w} \leq 0{ }^{11}$. It should be clear, thus, that we do not depart trom the most standard economic assumptions made on the topic but simply propose an arbitrarily detailed description of the content of the employee's utility function ${ }^{12}$.

\subsection{Psychological assumptions}

The choice of an identity by a working person reflects that of some particular context of self-evaluation. ${ }^{13}$ Considering an agent having identity $A$ (respectively $B$ ) comes to the case of an individual who views the occupational field (respectively some extraoccupational fields) as a context of selfevaluation. It conditions the motives for an agent's behavior in general, one's motivation within the occupational framework in particular. Below, we are interested in the way work motivation responds to employment terms offered by employers: this drives us to let the agent adjusts his identity to employment terms.

$a(w, e)$ must be considered as exhibiting little variation from one individual to the other. As far function $a($.$) is considered, its arguments w$ and $e$ play less as such than through what they mean to an agent having identity $A$. The very notion of an identity choice involves a willingness of signalling (identity for others) and auto-signalling (identity for oneself). ${ }^{14}$ Here, we provide a reduced form model of identity motives. All other things remaining equal, a higher wage whips up the self-esteem of a working person having identity $A, a_{w}^{\prime}>0$, as a positive signal (to others in particular) as regards his professional worth; a higher effort whips up his self-esteem, $a_{e}^{\prime}>0$, as a positive signal (to himself in particular) as regards his professional commitment ${ }^{15}$. These assumptions are meant to capture the notion of a non pecuniary work motivation: an agent having identity $A$ develops a non pecuniary work motivation, whereas one having identity $B$ does not.

10 When a property holds for any value of a function's argument, we omit to specify this argument. For instance, $f_{w}^{\prime}>0$ stands for $\forall(w, e, x) \in R_{+}^{3}, f_{w}^{\prime}(w, e, x)>0$.

11 Full description of what this involves as for function $f($.$) is provided in the appendix.$

12 Of course, the assumptions above are sufficient conditions for equilibrium existence; they are by no means necessary. The conditions to equilibrium existence is not an issue here.

13 See Gecas and Seff (1990).

14 See Bénabou and Tirole (2006).

15 For some references about the former assumption, see the social status literature as surveyed in Fershtman and Weiss (1993). As regards the latter assumption, see Kaufman (1999, p. 369). 
What about the way self-esteem amounts attached to polar identities $A$ and $B$ combine to determine $x^{\mathrm{x}}($.$) ? A sufficient condition to obtain the$ results of this paper is to assume that, for all $x \in] 0,1\left[, x^{\mathrm{x}}()<.\max \{a(), b\right.$.$\} .$ This comes to favour the idea that self-esteem requires specialization i.e. the choice of an exclusive context of self-evaluation. Although this assumption makes our reasoning much clearer, it is not a necessary condition.

In the remaining of the paper, for the sake of clarity, we will abuse our notations, writing $u^{A}(w, e)$ and $u^{B}(w, e)$ respectively for $u^{1}(w, e)$ and $u^{0}(w, e)$. Figure 1 illustrates how our identity scenario permits to deal with matters of non pecuniary work motivation. Two indifference curves (corresponding to a similar utility level $\underline{u}$ ) are depicted, corresponding to identities $A$ or $B$. For any $\underline{w}>\tilde{w}$, as compared to someone having identity $B$, an agent having identity $A$ exerts some additional (costless) effort. This additional effort responds to a non pecuniary work motivation.

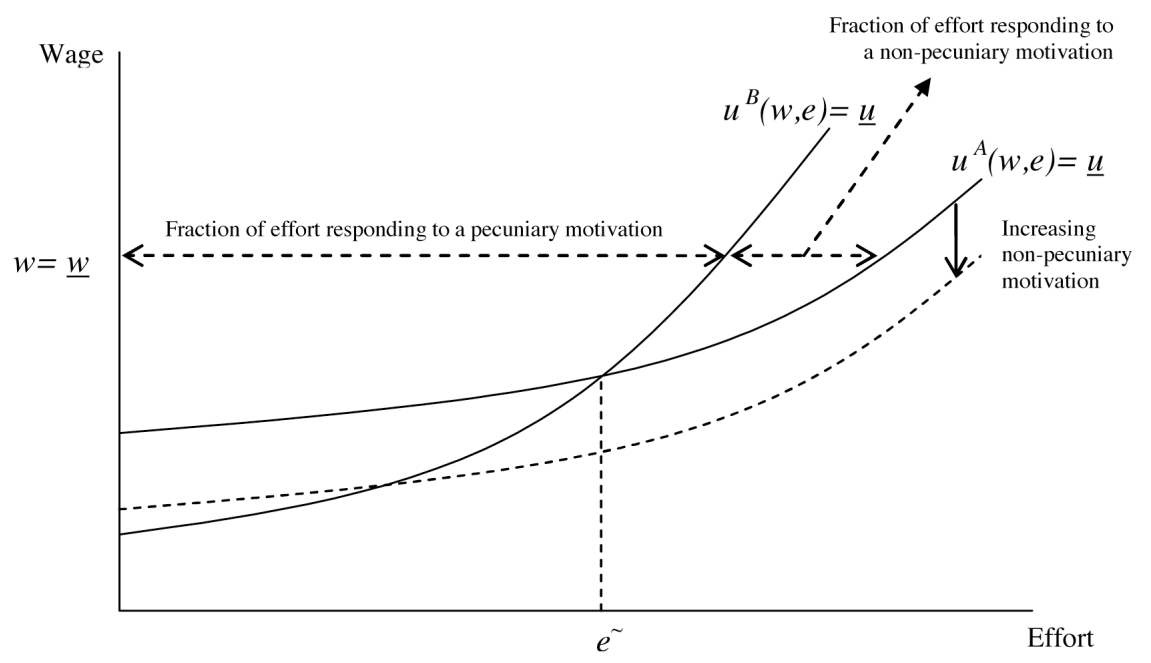

Figure 1: Identity-dependent indifference curve and non pecuniary work motivation.

\subsection{Timing}

The timing of the contracting game is as follows. (0) The characteristics of the job are observed both by the principal and the agent. (1) The principal offers employment terms consisting in a pair $(w, e):$ she commits to transferring $w \geq 0$ if the actual effort exerted by the agent is higher or equal to $e$. (2) The agent decides whether to accept the job, adopts an identity (chooses $\mathrm{x}$ ), and chooses his level of effort. (3) The contract is executed. Note that employment terms cannot be made contingent upon the agent's identity (the latter is assumed not to be verifiable). Given the principal offer 
$(w, e)$, if the agent accepts the job, chooses the identity combination $\mathbf{x}$, and exerts the actual effort $e_{a c t} \geq 0$, players' payoffs are $\left(S\left(e_{a c t}\right)-w, u^{\mathrm{x}}\left(w, e_{a c t}\right)\right)$ if $e_{a c t} \geq e$, and $\left(S\left(e_{a c t}\right), u^{\mathrm{x}}\left(0, e_{a c t}\right)\right)$ if $e_{a c t}<e$, whereas if the agent refuses the job, and chooses the combination $\mathrm{x}$, players' payoffs are $\operatorname{simply}\left(S_{\theta}, u_{\text {out }}^{\mathrm{x}}\right)$. In the remaining, the focus is on jobs which are actually filed in equilibrium. $S_{\theta}$ will thus always be assumed low enough so that the job be not left vacant in equilibrium. The most important aspect in this modeling is that the employer has the primary part (by defining employment terms) not only as regards the effort exerted by the agent but also as the subjective relation he has to his job is considered.

\subsection{The problem of the principal}

Clearly, under previous assumptions, $e_{a c t}>e$ cannot be a dominant strategy, while if $e_{a c t}<e$ then $e_{a c t}=0$. Assuming that she decides to hire the agent, the problem of the principal can thus be written as follows

$$
\begin{aligned}
& \max _{e, w \geq 0} S(e)-w \\
& \text { s.t. }\left\{\begin{array}{ll}
\max _{\mathrm{x}} u^{\mathrm{x}}(w, e) \geq \max _{\mathrm{x}} u^{\mathrm{x}}(0,0) & \text { (incentive condition) } \\
\max _{\mathrm{x}} u^{\mathrm{x}}(w, e) \geq \max _{\mathrm{x}} u_{\text {out }}^{\mathrm{x}} & \text { (participation condition) }
\end{array}\right. \text {. }
\end{aligned}
$$

We further assume that: for all $x \in] 0,1\left[, u_{\text {out }}^{A}<u_{\text {out }}^{\mathrm{x}}<u_{\text {out }}^{B}\right.$; for all $e \in R_{+}, u^{B}(0, e) \leq u_{o u t}^{B}$. The sine qua non for someone to draw self-esteem from one's job is obviously to have a job. That is what is meant with the former assumption: the more an outsider weights identity $A$ the lower his well-being. The latter assumption means that, for someone having identity $B$, being employed does not provide well-being in itself: it is always better being an outsider than a working-poor.

\section{Optimal contracts}

$S($.$) is defined and twice differentiable on R_{+}$. It is assumed that: for all $e \in R_{+}, S^{\prime}(e)>0$ and $\lim _{e \rightarrow 0} S^{\prime}(e)=+\infty$; for all $e \in R_{+}, S^{\prime \prime}(e)<0$. Let us define $\omega^{X}(),. X \in\{A, B\}$, for all $e \geq 0$, by $u^{X}\left(\omega^{X}(e), e\right)=\underline{u}$ where $\underline{u}=\max \left\{u^{A}(0,0), u_{\text {out }}^{B}\right\}$. Transfer $\omega^{X}(e)$ is the amount the principal should pay to obtain effort $e$ from the agent while inducing identity $X$. Let $(\tilde{\omega}, \tilde{e})$ be defined by $\tilde{w}=\omega^{A}(\tilde{e})=\omega^{B}(\tilde{e})$. ( $\left.\tilde{w}, \tilde{e}\right)$ represents an endogenous benchmark as regards agent's choice of an identity. For $\tilde{e} \geq 0,(\tilde{w}, \tilde{e})$ are the only employment terms leaving the agent indifferent between identities $A$ and $B$. Therefore any employment terms $(w, e) »(\tilde{w}, \tilde{e})$ signal a genuine holder of the identity $A$. We denote $\left(w^{*}, e^{*}\right)$ the optimal contract. 
Lemma 1. Equilibrium exists, and $\left(w^{*}, e^{*}\right) \in\left\{\left(w^{A}, e^{A}\right),\left(w^{B}, e^{B}\right)\right\}$ where, for $X \in\{A, B\},\left(w^{X}, e^{X}\right)$ is such that $S^{\prime}\left(e^{X}\right)=\frac{d \omega^{X}}{d e}\left(e^{X}\right)$, and $w^{X}=\omega^{X}\left(e^{X}\right)$.

Proof. See the appendix.

We can thus distinguish between two types of jobs depending on the identity, $A$ or $B$, the optimal contract induces. For short, we will refer to them as jobs of type $A$, when involving a non pecuniary work motivation, or jobs of type $B$.

Proposition 2. For $\omega^{A}(0) \leq \omega^{B}(0)$, equilibrium does not depend on $S($. and $\left(w^{*}, e^{*}\right)=\left(w^{A}, e^{A}\right)$. This is not true anymore if $\omega^{A}(0)>\omega^{B}(0)$. In that case, $\tilde{e}$ exists, is positive, and: if $S^{\prime}(\tilde{e}) \geq \frac{d \omega^{B}}{d e}(\tilde{e})$ then $\left(w^{*}, e^{*}\right)=\left(w^{A}, e^{A}\right)$; if $S^{\prime}(\tilde{e}) \leq \frac{d \omega^{A}}{d e}(\tilde{e})$ then $\left(w^{*}, e^{*}\right)=\left(w^{B}, e^{B}\right)$; if $\frac{d \omega^{B}}{d e}(\tilde{e})>S^{\prime}(\tilde{e})>\frac{d \omega^{A}}{d e}(\tilde{e})$ then candidates $\left(w^{A}, e^{A}\right)$ and $\left(w^{B}, e^{B}\right)$ are such that $w^{A}>\tilde{w}>w^{B}$ and $e^{A}>\tilde{e}>e^{B}$.

Proof. See the appendix. $\min \left\{\omega^{A}(0), \omega^{B}(0)\right\}$ represents the lowest wage amount ensuring the agent participation. $\omega^{A}(0) \leq \omega^{B}(0)$ implies that this amount induces the identity $A$ which conveys a non pecuniary work motivation. We are dealing with jobs the characteristics of which always induce such an extra motivation. One can think to high prestige jobs: whatever the productivity of the job, the optimal contract induces a non pecuniary motivation. $\omega^{A}(0)>\omega^{B}(0)$ implies that the lowest wage amount ensuring participation induces identity $B$ i.e. fails to induce a non pecuniary motivation. When the optimal effort is low, it is better for the principal to simply give up virtual extra motivation. When this optimal effort is high, it is worth paying the agent above $\tilde{w}$ to induce an effort well over $\tilde{e}$. In this latter configuration, we first focus on most extreme cases (when productivity is particularly high or low) which are conclusive without further specifications. Starting from an effort $\tilde{e}$, the marginal cost of effort is always higher without extra work motivation (see lemma A5 in the appendix). The problem of the principal is therefore to decide between reducing costs against a lower output (inducing identity $B$ ) or increasing output against higher costs (inducing identity $A$ ). $S^{\prime}(\tilde{e}) \geq \frac{d \omega^{B}}{d e}(\tilde{e})$ means that costs reduction cannot offset the loss in output; furthermore, it ensures $S^{\prime}(\tilde{e}) \geq \frac{d \omega^{A}}{d e}(\tilde{e})$ i.e. that the gain in output attached to identity $A$ exceeds the increase in costs. The principal is better inducing 
identity $A$. The reasoning is symmetric for $\frac{d \omega^{A}}{d e}(\tilde{e}) \geq S^{\prime}(\tilde{e})$. As for cases in between, further specification is required to conclude. However, one can guarantee $\left(w^{A}, e^{A}\right) »(\tilde{w}, \tilde{e}) »\left(w^{B}, e^{B}\right)$ so that employment terms indeed signal the identity held by the agent. Hereafter, we focus on jobs the characteristics of which entail $\omega^{A}(0)>\omega^{B}(0)$.

\section{Jobs productivity and working conditions}

To further detail the analysis, it is useful to get equipped with some specific parameters. First, let $\gamma>0$ be defined by $S(e)=\gamma \hat{S}(e): \gamma$ is a global productivity parameter. It has an identical influence on productivity whatever the effort level considered. Note that $\gamma$ does not impact the curvature of the surplus function. Second, let $\psi>0$ denote a parameter of working conditions. It is assumed that $u^{\mathrm{x}}(w, e)=f\left(w, \frac{e}{\psi} ; x^{\mathrm{x}}(w, e)\right)$, so as to capture the idea that better working conditions reduce the disutility of effort.

For any fixed preferences such that $\omega^{A}(0)>\omega^{B}(0)$, one can define $\left(\underline{e}^{A}, \bar{e}^{B}\right)$ and $\tilde{\gamma}$ as solutions to:

$$
\left\{\begin{array}{l}
\frac{d \omega^{A}}{d e}\left(\underline{e}^{A}\right)=\tilde{\gamma} \hat{S}^{\prime}\left(\underline{e}^{A}\right) \\
\frac{d \omega^{B}}{d e}\left(\bar{e}^{B}\right)=\tilde{\gamma} \hat{S}^{\prime}\left(\bar{e}^{B}\right) \\
\tilde{\gamma} \hat{S}\left(\underline{e}^{A}\right)-\omega^{A}\left(\underline{e}^{A}\right)=\tilde{\gamma} \hat{S}\left(\bar{e}^{B}\right)-\omega^{B}\left(\bar{e}^{B}\right)
\end{array} .\right.
$$

Similarly, for a given surplus function, and some working conditions $\psi$, one can define $\left(\underline{e}^{A}, \bar{e}^{B}\right)$ and $\psi$ as solutions to:

$$
\left\{\begin{array}{l}
\frac{d \omega^{A}}{d e}\left(\underline{e}^{A} ; \tilde{\psi}\right)=S^{\prime}\left(\underline{e}^{A}\right) \\
\frac{d \omega^{B}}{d e}\left(\bar{e}^{B} ; \tilde{\psi}\right)=S^{\prime}\left(\bar{e}^{B}\right) \\
S\left(\underline{e}^{A}\right)-\omega^{A}\left(\underline{e}^{A} ; \tilde{\psi}\right)=S\left(\bar{e}^{B}\right)-\omega^{B}\left(\bar{e}^{B} ; \tilde{\psi}\right)
\end{array} .\right.
$$

In both cases, with well-behaved functions posited above, $\left(\underline{e}^{A}, \bar{e}^{B}\right)$ and $\tilde{\gamma}$ (respectively, $\tilde{\psi}$ ) exist and are unique. The pair $\left(\underline{w}^{A}, \underline{e}^{A}\right)$ represents the lowest employment terms inducing a non pecuniary work motivation; $\left(\bar{w}^{B}, \bar{e}^{B}\right)$ the highest employment terms an agent with identity $B$ may obtain. Note that $\bar{e}^{B}<\tilde{e}<\underline{e}^{A}$. 


\subsection{Jobs productivity}

When preferences are fixed, marginal productivity takes a crucial part in determining the type $(A$ or $B$ ) of some particular job. For $S(e)=\hat{\gamma} \hat{S}(e)$, the principal is indifferent between the two contracts.

Proposition 3. For $\omega^{A}(0)>\omega^{B}(0)$, the optimal contract is

$$
\left(w^{*}, e^{*}\right)= \begin{cases}\left(w^{B}, e^{B}\right) & \text { if } \gamma \leq \tilde{\gamma} \\ \left(w^{A}, e^{A}\right) & \text { if } \gamma \geq \tilde{\gamma}\end{cases}
$$

Proof. This directly derives from our writings: $\gamma<\tilde{\gamma} \Leftrightarrow \gamma \hat{S}\left(e^{A}\right)-\omega^{A}\left(e^{A}\right)$ $<\hat{\gamma}\left(e^{B}\right)-\omega^{B}\left(e^{B}\right) \Leftrightarrow \pi^{A}<\pi^{B}$ and thus $\left(w^{*}, e^{*}\right)=\left(w^{B}, e^{B}\right)$.

The gap $\underline{w}^{A}-\bar{w}^{B}$ represents a fixed cost corresponding to the price of a non pecuniary work motivation. For such a fixed cost to be worth bearing, optimal effort $e^{A}$ must be high enough. Increasing $\gamma$ comes to increasing optimal effort and to make it worthwhile inducing an extra motivation. This is basically an increasing return to scale mechanism. The non-convexity of indifference curves introduces a leap in employment terms corresponding to a switch in human ressources management strategy.

\subsection{Working conditions}

Let $\tilde{\rho}=\frac{S^{\prime}\left(\bar{e}^{B}\right) \bar{e}^{B}}{S^{\prime}\left(\underline{e}^{A}\right) \underline{e}^{A}}: \tilde{\rho}$ represents a coefficient of curvature of $S($.$) over the$ interval $\left[\bar{e}^{B}, \underline{e}^{A}\right]$ : the higher $\tilde{\rho}$ the higher the reduction in marginal productivity between $\bar{e}^{B}$ and $\underline{e}^{A}$.

Proposition 4. For $\omega^{A}(0)>\omega^{B}(0)$,

if $\tilde{\rho}<\left(1-\left|\frac{f_{x}^{A^{\prime}} a_{e}^{A_{e}^{\prime}}}{f_{e}^{A^{\prime}}}\right|\right)^{-1}$, equilibrium is given by $\left(w^{*}, e^{*}\right)=\left\{\begin{array}{ll}\left(w^{B}, e^{B}\right) & \text { if } \psi \leq \tilde{\psi} \\ \left(w^{A}, e^{A}\right) & \text { if } \psi \geq \tilde{\psi}\end{array}\right.$;

if $\tilde{\rho}>\left(1-\left|\frac{f_{x}^{A^{\prime}} a_{e}^{A^{\prime}}}{f_{e}^{A^{\prime}}}\right|\right)^{-1}$, equilibrium is given by $\left(w^{*}, e^{*}\right)= \begin{cases}\left(w^{B}, e^{B}\right) & \text { if } \psi \geq \tilde{\psi} \\ \left(w^{A}, e^{A}\right) & \text { if } \psi \leq \tilde{\psi}\end{cases}$

with following shortwritings: $f_{\tilde{x}}^{A^{\prime}}=f_{x}^{\prime}\left(\underline{w}^{A}, \frac{e_{\tilde{\psi}}^{A}}{\tilde{\psi}}, a\left(\underline{w}^{A}, \underline{e}^{A}\right)\right), f_{\bar{e}}^{A^{\prime}}=f_{e}^{\prime}\left(\underline{w}^{A}, \frac{e_{\tilde{\psi}}^{A}}{\tilde{\psi}}, a\left(\underline{w}^{A}, \underline{e}^{A}\right)\right)$, and $a_{e}^{\underline{A}}{ }^{\prime}=a_{e}^{\prime}\left(\underline{w}^{A}, \underline{e}^{A}\right)$.

Proof. See the appendix.

The threshold $\left(1-\left|\frac{f_{x}^{A^{\prime}} a_{e}^{A_{e}^{\prime}}}{f_{e}^{A^{\prime}}}\right|\right)^{-1}>0$ negatively depends on the relative weights of standard disutility versus self-esteem motives attached to effort 
in $\left(\underline{w}^{A}, \underline{e}^{A}\right)$. Let us consider preferences as fixed and discuss the role of $\tilde{\rho}$. The level of $\tilde{\rho}$ conditions the best response of the principal to a variation of $\psi$. From the reference situation $\psi$, an improvement in working conditions (an increase in $\psi$ ) reduces the marginal cost of effort (for any effort level). This drives the principal to reduce wage and to increase effort requirements all other things being equal. Depending on the form of the surplus function, the main response will be either to reduce costs or to increase output. For $\tilde{\rho}<\left(1-\left|\frac{f_{x}^{A^{\prime}} a_{e}^{A_{e}^{\prime}}}{f_{e}^{A^{\prime}}}\right|\right)^{-1}$, marginal productivity decreases slowly as effort increases and the main response is the latter (output increasing) for which an extra motivation is welcome (identity $A$ has a comparative advantage over $B)$. For $\tilde{\rho}>\left(1-\left|\frac{f_{x}^{A^{\prime}} e_{e}^{A^{\prime}}}{f_{e}^{A^{\prime}}}\right|\right)^{-1}$, marginal productivity decreases sharply as effort increases and the main response is cost cutting: on that ground, identity $B$ has a comparative advantage over $A$. The higher the relative weight of self-esteem motives as compared to standard disutility of effort - the higher $\left(1-\left|\frac{f_{x}^{A^{\prime}} a_{e}^{A^{\prime}}}{f_{e}^{A^{\prime}}}\right|\right)^{-1}$ - the more likely the former response. To sum up, the link between working conditions and non pecuniary work motivation depends on the chief concern of the employer. If this is expansion, non pecuniary work motivation should go with better working conditions; conversely, if this is cost cutting, better working conditions are attached to a purely pecuniary motivation.

\section{$5 \quad$ Male-female disparities in the labour market}

Let us come to the applied part of the analysis and tackle the problem of male-female disparities in the labour market. In this section, we use the model presented above to capture the role of non pecuniary work motivation in male-female disparities as suggested by empirical investigations.

\subsection{Male-female differences}

Workers are of type $\theta \in\{0,1\}$ subscript 0 refering to women, subscript 1 to men. We assume $b_{0}>b_{1}$ i.e. that, for some reason, female workers have more opportunity than male to enjoy self-esteem outside work, all other things being equal. Let's say that because of a socially appreciated maternal role, female have greater opportunities than male to enjoy self-esteem outside work i.e. that outside-work is a context of self-evaluation more favorable to women than to men. A direct consequence of this assumption is that men are never hired in jobs of type $B$. Figure 2 illustrates this point. It depicts: (a) identity-dependent indifference curves of a male worker 
$\left(u_{1}(w, e)=\underline{u}\right)^{16}$ on the one hand, of a female worker $\left(u_{0}(w, e)=\underline{u}\right)^{17}$ on the other hand (when it is optimal both to male and female to have identity $A$, curves merge); (b) iso-profit curves $(S(e)-w=\pi)$. Curves relating to the male worker are dotted. The profit of the principal is the highest when inducing identity $B$, in which case, it is better to hire a female worker. Employment terms of such a job cannot be accepted by a male worker.

However, both men and women may hold a job of type $A$, as figure 3 illustrates. Here, employment terms proposed by the employer induce a non pecuniary motivation (identity $A$ ) and are thus acceptable to both gender.

In the remaining, we consider labour market equilibria. There are $n$ workers of each gender. These workers distribute into $2 n$ jobs indexed by $j \in\{1,2, \ldots, 2 n\}$. Jobs differ in many dimensions and, virtually, a highly paid job may be of type $B$. Therefore, it is not clear how male and female workers should distribute between highly and poorly paid jobs, high and low effort-intensive jobs. Below we propose to study the specific impact of parameters introduced so far which are correlated to the type of jobs ( $A$ or $B)$, and eventually, to wage and effort.

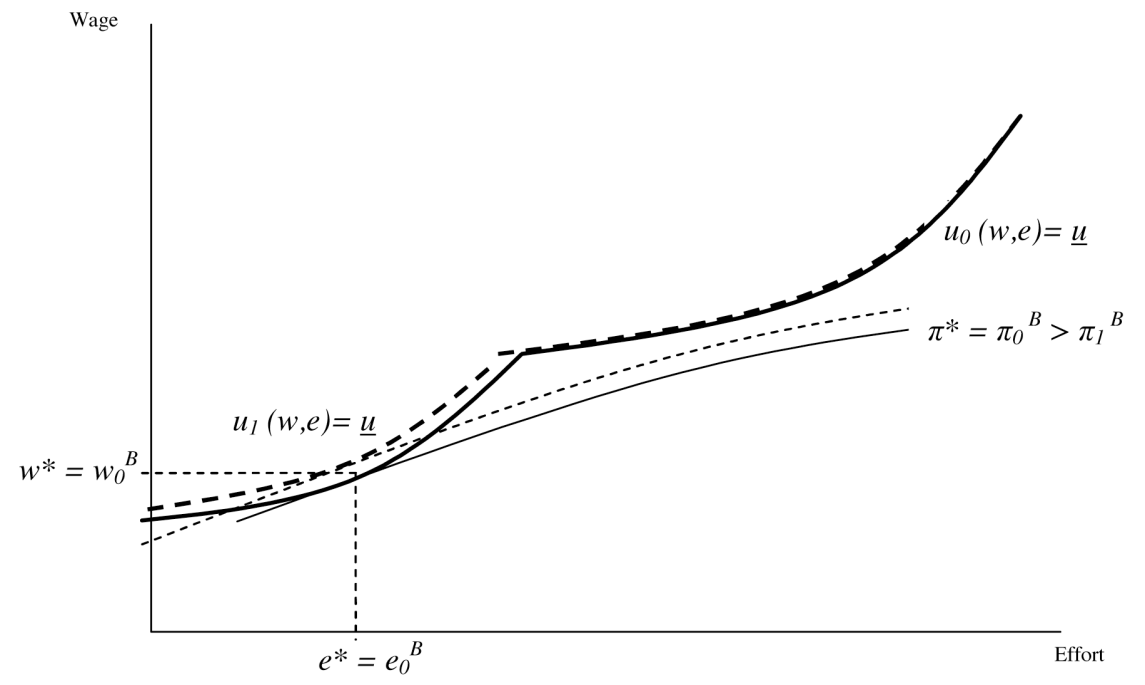

Figure 2: Female workers accept employment terms (inducing the identity B) that a male worker would not accept.

$$
\begin{aligned}
& 16 \quad u_{1}(w, e)=\max \left\{u_{1}^{A}(w, e), u_{1}^{B}(w, e)\right\} . \\
& 17 \quad u_{0}(w, e)=\max \left\{u_{0}^{A}(w, e), u_{0}^{B}(w, e)\right\} .
\end{aligned}
$$




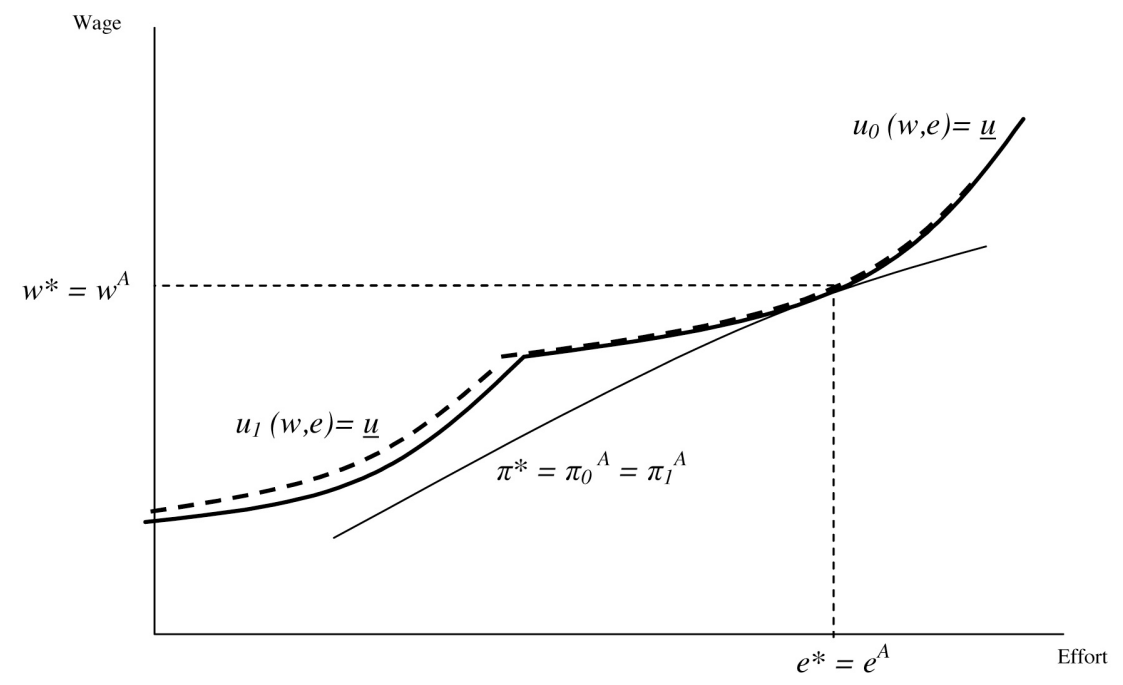

Figure 3: Employment terms offered by the employer are acceptable both to male and female workers.

\subsection{Male-female disparities in jobs productivity}

Suppose jobs only differ according to their productivity as captured by parameter $\gamma$. Jobs are indexed by $j$ so that the productivity of job $j$ is denoted $\gamma_{j}$. Depending on this parameter, jobs will be of type $A$ or $B$. The higher parameter $\gamma_{j}$, the more likely job $j$ will be of type $A$ as proposition 3 shows. We are concerned with male and female workers distributions over those jobs. Previous analysis guarantees that there will be no male workers in jobs of type $B$, but it does not rule out that some female workers be employed in jobs of type $A$. Eventually, male-female differences depend on how female workers distribute over varously productive jobs of type $A$. We treat this distribution as random and provide results holding in expectation. Hereafter, we take every gendered employment profiles over jobs of type $A$ as equally likely and consider expected values of average productivity, wage and effort in jobs held by female workers on the one hand, male workers, on the other hand.

Corollary 5. The expected average productivity of jobs held by male workers is higher than that of jobs held by female workers. Furthermore, the gap in expected average productivity is increasing in the number of jobs of type $B$.

Proof. See the appendix. 
Corollary 6. The expected average effort exerted by male workers is higher than that exerted by female workers. So are expected average earnings respectively of male and female workers.

Proof. This directly derives from previous corrolary. For $X \in\{A, B\}$, the optimal effort exerted on some job $j$ of type $X, e^{X}\left(\gamma_{j}\right)$ is defined by $\frac{d \omega^{X}}{d e}\left(e^{X}\left(\gamma_{j}\right)\right)=\gamma_{j} \hat{S}^{\prime}\left(e^{X}\left(\gamma_{j}\right)\right)$ with $\frac{d \omega^{X}}{d e}($.$) strictly increasing and$ $\hat{S}^{\prime}($.$) strictly decreasing in e$. It results that $e^{X}$ is strictly increasing in $\gamma_{j}$ and so is $w^{X}=\omega^{X}\left(e^{X}\right)$.

\subsection{Male-female disparities in working conditions}

We can conduct a similar analysis for jobs varying according to working conditions as captured by $\psi_{j}$. The reference is then the proposition 4 and two classes of jobs should be distinguished depending on the main concern of the employer (increasing output or reducing costs). Let output oriented jobs be defined by $\tilde{\rho}<\left(1-\left|\frac{f_{x}^{A^{\prime}} a^{A^{\prime}}}{f_{e}^{A^{A}}}\right|\right)^{-1}$, and cost cutting oriented jobs by $\tilde{\rho}>\left(1-\left|\frac{f_{x}^{A^{\prime}} a_{e}^{A^{\prime}}}{f_{e}^{A^{\prime}}}\right|\right)^{-1}$.

Corollary 7. Consider the class of output oriented jobs. The expected average working conditions of male workers are better than that of female workers. Furthermore, the gap in expected average working conditions is increasing in the number of jobs of type $B$.

Corollary 8. Consider the class of cost cutting oriented jobs. The expected average working conditions of female workers are better than that of male workers. Furthermore, the gap in expected average working conditions is increasing in the number of jobs of type $B$.

Proof. The last two corrolaries exploit proposition 4 in the same way as previous ones exploit proposition 3: the reasoning is similar.

The link between male-female disparities in working conditions and male-female earnings disparities is less direct than in the case of malefemale disparities in jobs productivity. However, there are good reasons to think that male-female disparities in working conditions will fuel the malefemale earnings gap. We illustrate our point in figures 4 (devoted to output oriented jobs) and 5 (devoted to cost cutting oriented jobs). Each figure depicts three jobs: one job of reference (Ref), one with poorer working conditions than the reference, one with better working conditions than the reference. In both cases, it is sufficient to depict the indifference cuves of female workers. Consider the class of output oriented jobs. Jobs with poor working conditions (as compared to the reference) will be exclusively held by female workers exerting effort $e_{0}^{B}$ and earning $w_{0}^{B}$. From an argument 
similar to that of the previous section, male workers should be overrepresented in output oriented jobs with good working conditions, exerting effort $e^{A}$ and earning $w^{A}$. As the figure 4 illustrates, the gap between $w_{0}^{B}$ and $w^{A}$ should be small (if positive). Let us turn to the class of cost cutting oriented jobs. In this latter case, the overrepresentation of male workers in jobs with poor working conditions should contribute a lot to the malefemale earnings gap as $w^{A}$ is well above $w_{0}^{B}$. In one case (that of output oriented jobs), better working conditions enjoyed by male as compared to female contribute little to wage differentials; in the other case (that of cost cutting oriented jobs), better working conditions enjoyed by female as compared to male contribute a lot to wage differentials (in favor of male workers). Overall, in our model, working conditions do not have a symmetric role as far male and female workers are concerned.

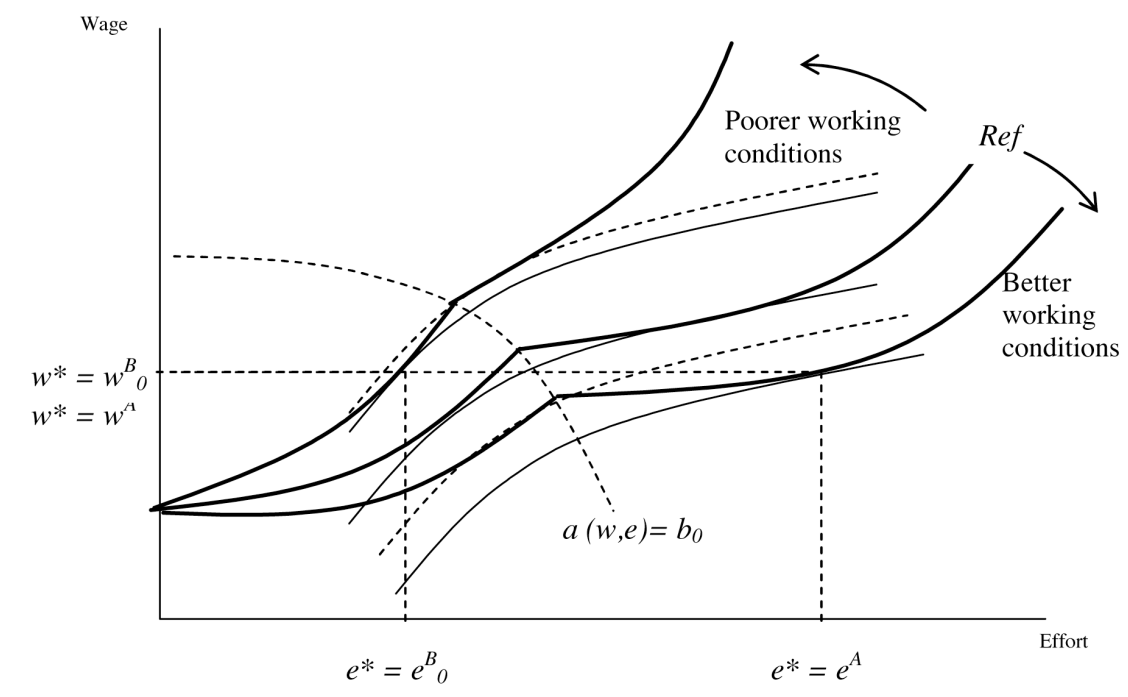

Figure 4: Output oriented jobs: $\tilde{\rho}<\left(1-\left|\frac{f_{x}^{A^{\prime}} e_{e}^{A^{\prime}}}{f_{e}^{A^{\prime}}}\right|\right)^{-1}$. Improved working conditions drives the principal to expand effort requirement with little variation in costs.

\section{Conclusion}

In the analysis above, we have provided a reduced form model of non pecuniary work motivation as resulting from adequate employment terms. Jobs are characterized by two dimensions, productivity and working conditions, which influence employers human ressources management strategies (inducing an extra work motivation or not). We have shown that, all other things 
being equal, high productivity jobs are more likely to drive the employer to offer employment terms inducing a non pecuniary work motivation. The impact of working conditions on the employer strategy depends of the main concern attached to the job under consideration: cost cutting versus output expanding. In cost cutting oriented jobs, extra work motivation go with poor working conditions; in output expanding oriented jobs, extra work motivation go with good working conditions.

This model is applied to the issue of male-female disparities in the labor market to account for dimensions pointed up by empirical studies but hardly captured in standard models of employment relation: work attitudes. The basic assumption is that female enjoy better opportunity to draw self-esteem from the outside work than male workers. This implies that female may accept employment terms disregarding non pecuniary work motivation whereas male workers do not. We can then study the distribution of female workers, on the one hand, male workers, on the other hand, over various jobs. These jobs vary according to productivity, working conditions, and eventually, employment terms (wage and effort requirement). We show that female are overrepresented in low effort requirement poorly paid jobs. As a consequence, the average male wage is expected to be higher than that of female.

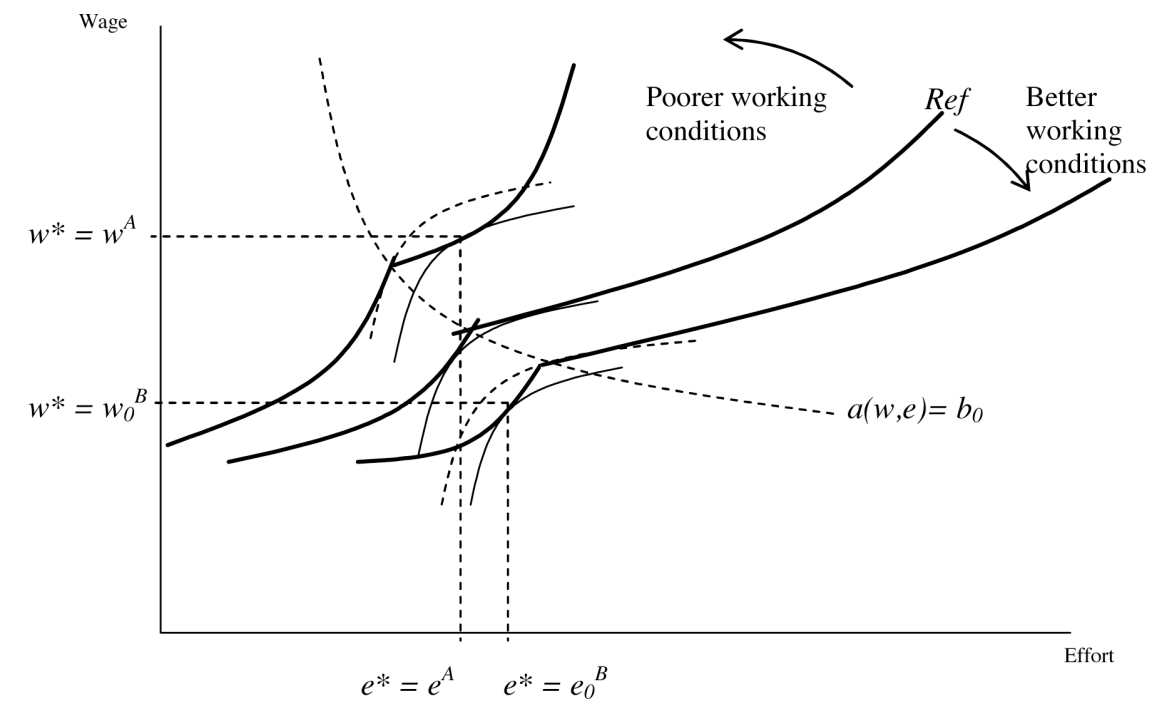

Figure 5: Cost cutting oriented jobs: $\tilde{\rho}>\left(1-\left|\frac{f_{x}^{A^{\prime}} a_{e}^{\frac{A^{\prime}}{A^{\prime}}}}{f^{A^{\prime}}}\right|\right)^{-1}$. Improved working conditions drives the principal to reduce costs whth little varıation in effort requirements. 


\section{References}

[1] Akerlof G.A. et R.E. Kranton (2000). "Economics and Identity", Quarterly Journal of Economics, CXV, pp.715-753.

[2] Akerlof G.A. et R.E. Kranton (2005). "Identity and the Economics of Organizations", Journal of Economic Perspectives, vol. 19, n 1, pp.9-32.

[3] Blau F. D. et L.M. Kahn (2000). "Gender Differences in Pay", Journal of Economic Perspectives, vol. 14, $\mathrm{n}^{\circ} 14$, pp.75-99.

[4] Benabou R. et J. Tirole (2006). "Incentives and Prosocial Behavior", American Economic Review, vol. 96, n ${ }^{\circ}$ 5, pp. 1652-1678.

[5] Frey B. (1997). Not Just for the Money. An Economic Theory of Personal Motivation, Cheltenham: Edward Elgar.

[6] Fehr E. et A. Falk (2002). "Psychological foundations of incentives", European Economic Review, vol. 46, n $4-5$, pp.687-724.

[7] François P. (1998). "Gender discrimination without gender difference: theory and policy responses", Journal of Public Economics, vol. 68, no 1, pp.1-32.

[8] Gecas V. et M.A. Seff (1990). "Social class and self-esteem: Psychological centrality, compensation, and the relative effects of work and home", Social Psychology Quarterly, vol. 53, n 2, pp.165-173.

[9] Hakim C. (2003). "A New Approach to Explaining Fertility Patterns: Preference Theory", Population and Development Review, vol. 29, n 3, pp.349-374.

[10] Johnson G. et G. Solon (1986). "Estimates of the Direct Effects of Comparable Worth Policy", American Economic Review, vol. 76, n 5, pp.1117-25.

[11] Kaufman E.B., (1999). "Expanding the Behavioral Foundations of Labor Economics", Industrial and Labor Relations Review, vol. 52, n 3, pp.361-392.

[12] Lambert S.J. (1991). "The combined effects of job and family characteristics on the job satisfaction, job involvement, and intrinsic motivation of men and women workers", Journal of Organizational Behavior, vol.12, pp.341-363.

[13] Macpherson D.A. et B.T. Hirsch (1995). "Wages and Gender Composition: Why do Women's Jobs Pay Less?", Journal of labor Economics, vol.13, n 3 , pp.426-471.

[14] OCDE (2002). "Women at work: who are they and how are they faring?", OECD Employment Outlook, pp.61-125.

[15] Fershtman C. et Y. Weiss (1993). "Social Status, Culture and Economic Performance", The Economic Journal, vol. 103, no 419, pp.946-959. 


\section{A Appendix}

What standard conditions on $u^{X}($.$) involve on f($.$) and x^{X}($.$) .$

$$
\begin{aligned}
& \frac{\partial u^{\mathrm{x}}}{\partial w}=f_{>0}^{\prime}+\underbrace{f_{x}^{\prime}}_{>0} \underbrace{x_{w}^{\prime}}_{\geq 0}>0 \\
& \frac{\partial u^{\mathrm{x}}}{\partial e}=f_{<0}^{f_{e}^{\prime}}+\underbrace{f_{x}^{\prime} x_{e}^{\prime}}_{>0}<0 \\
& \frac{\partial^{2} u^{\mathrm{x}}}{\partial w^{2}}=f_{\substack{w^{2} \\
\text { In }}}^{\prime \prime}+2 f_{w x^{\prime \prime}}^{\prime \prime} x_{w}^{\prime}+f_{x^{2}}^{\prime \prime}\left(x_{w}^{\prime}\right)^{2}+f_{x}^{\prime} x_{w^{2}}^{\prime \prime} \leq 0 \\
& \frac{\partial^{2} u^{\mathrm{x}}}{\partial w \partial e}=f_{\substack{\leq e \\
\leq 0}}^{\prime \prime}+f_{w x}^{\prime \prime} x_{e}^{\prime}+\left(f_{x e}^{\prime \prime}+f_{x^{2}}^{\prime \prime} x_{e}^{\prime}\right) x_{w}^{\prime}+f_{x}^{\prime} x_{w e}^{\prime \prime} \leq 0 \\
& \frac{\partial^{2} u^{\mathrm{x}}}{\partial e^{2}}=f_{\substack{e^{2} \\
\leq 0}}^{\prime \prime}+2 f_{e x}^{\prime \prime} x_{e}^{\prime}+f_{x^{2}}^{\prime \prime}\left(x_{e}^{\prime}\right)^{2}+f_{x}^{\prime} x_{e^{2}}^{\prime \prime} \leq 0
\end{aligned}
$$

\section{A.1 Optimal contracts}

Let $u(w, e)=\max \left\{u^{A}(w, e), u^{B}(w, e)\right\}$.

Lemma $\mathbf{A} 1$ The problem of the principal writes $\max _{e, w \geq 0} S(e)-w$ s.t. $u(w, e) \geq \underline{u}$.

Proof. We show first that $\max _{\mathrm{x}} u^{\mathrm{x}}(w, e)=u(w, e)$. Take $\left.x \in\right] 0,1[$. For all $(w, e) \in R_{+}^{2}$ such that $a(w, e)<b, \max \left\{u^{A}(w, e), u^{\mathrm{x}}(w, e)\right\}<u^{B}(w, e)$; for all $(w, e) \in R_{+}^{2}$ such that $a(w, e)=b, u^{A}(w, e)=u^{B}(w, e)>u^{\mathrm{x}}(w, e)$; for all $(w, e) \in R_{+}^{2}$ such that $a(w, e)>b, u^{A}(w, e)>\max \left\{u^{\mathrm{x}}(w, e), u^{B}(w, e)\right\}$. Thus, for all $(w, e) \in R_{+}^{2}, \max _{x \in[0,1]} u^{x}(w, e)=\max \left\{u^{A}(w, e), u^{B}(w, e)\right\}$ $=u(w, e)$. Let us prove, now, that $\max \left\{\max _{\mathrm{x}} u^{\mathrm{x}}(0,0), \max _{\mathrm{x}} u_{\text {out }}^{\mathrm{x}}\right\}=\underline{u}$. Our assumptions directly lead to $\max _{\mathrm{x}} u^{\mathrm{x}}=u_{\text {out }}^{B}$ while $\max _{\mathrm{x}} u^{\mathrm{x}}(0,0)$ $=\max \left\{u^{A}(0,0), u^{B}(0,0)\right\}$ derives from previous step of the proof. Consequently the participation condition writes $u(w, e) \geq \max \left\{\max \left\{u^{A}(0,0)\right.\right.$, $\left.\left.u^{B}(0,0)\right\}, u_{\text {out }}^{B}\right\}$ with

$$
\max \left\{\max \left\{u^{A}(0,0), u^{B}(0,0)\right\}, u_{\text {out }}^{B}\right\}=\max \left\{u^{A}(0,0), u^{B}(0,0), u_{\text {out }}^{B}\right\}
$$

and, since by assumption $u^{B}(0,0) \leq u_{\text {out }}^{B}, \quad \max \left\{u^{A}(0,0), u^{B}(0,0), u_{\text {out }}^{B}\right\}=$ $\max \left\{u^{A}(0,0), u_{\text {out }}^{B}\right\}=\underline{u}$. 
Lemma A2 In equilibrium, the constraint is binding $u\left(w^{*}, e^{*}\right)=\underline{u}$.

Proof. Suppose $u\left(w^{*}, e^{*}\right)>\underline{u}$ and consider the pair of positive deviations $(d w, d e)$ (which exists), defined by $u\left(w^{*}-d w, e^{*}+d e\right)=\underline{u}$. Clearly, $S^{\prime}\left(e^{*}\right) d e-\left(w^{*}-d w\right)>0$ which implies that $\left(w^{*}, e^{*}\right)$ is not an optimum, a contradiction.

\section{A.1.1 Identity dependent indifference curves}

Lemmas below present some useful results on the shape of indifference curves.

Lemma A3 The next statements hold: for $X \in\{A, B\}, \omega^{\mathrm{x}}(0) \geq 0$ and $\omega^{\mathrm{x}}($. is strictly increasing in $e$; for all $e \geq 0, \omega^{A}(e)>\omega^{B}(e) \Leftrightarrow a\left(\omega^{A}(e), e\right)<b$.

Proof. The first item is standard: for $X \in\{A, B\}, u^{\mathrm{x}}\left(\omega^{\mathrm{x}}(e), e\right)=$ $\underline{u} \Rightarrow \frac{d \omega^{\mathrm{X}}}{d e}(e)=-\frac{\frac{\partial u^{X}}{\partial e}\left(\omega^{X}(e), e\right)}{\frac{\partial u^{X}}{\partial w}\left(\omega^{X}(e), e\right)}, \frac{\partial u^{X}}{\partial e}<0$, and $\frac{\partial u^{X}}{\partial w}>0$ entails $\frac{\partial \omega^{X}}{\partial e}>0$, hence the conclusion. Concerning the second item, let us first recall that $f_{w}^{\prime}$, $f_{x}^{\prime}, a_{w}^{\prime}$ and $a_{e}^{\prime}$ are all strictly positive. For all $e \geq 0, \omega^{A}(e)>\omega^{B}(e)$ implies $f\left(\omega^{B}(e), e, b\right)<f\left(\omega^{A}(e), e, b\right)$ but $f\left(\omega^{B}(e), e, b\right)=\underline{u}=f\left(\omega^{A}(e), e, a\left(\omega^{A}(e), e\right)\right)$ so that $f\left(\omega^{A}(e), e, a\left(\omega^{A}(e), e\right)\right)<f\left(\omega^{A}(e), e, b\right)$. Since $f_{x}^{\prime}>0$, it requires $a\left(\omega^{A}(e), e\right)<b$. For all $e \geq 0, a\left(\omega^{A}(e), e\right)<b$ implies $f\left(\omega^{A}(e), e, a\left(\omega^{A}(e), e\right)\right)<$ $f\left(\omega^{A}(e), e, b\right)$ but $f\left(\omega^{A}(e), e, a\left(\omega^{A}(e), e\right)\right)=\underline{u}=f\left(\omega^{B}(e), e, b\right)$ so that $f\left(\omega^{B}(e), e, b\right)<f\left(\omega^{A}(e), e, b\right)$. Since $f_{w}^{\prime}>0$, it requires $\omega^{A}(e)>\omega^{B}(e)$.

Let us define $\tilde{\omega}($.$) , for all e$, by $a(\tilde{\omega}(e), e)=b$. Note that $\tilde{\omega}(\tilde{e})=\omega^{A}(\tilde{e})=\omega^{B}(\tilde{e})$.

Lemma A4 If $\omega^{A}(0)>\omega^{B}(0)$ then there exists $\tilde{e}>0$ such that, for all $e \leq \tilde{e}$, $\omega^{B}(e) \leq \omega^{A}(e)$ and, for all $e>\tilde{e}, \omega^{B}(e)>\omega^{A}(e)$. If $\omega^{B}(0) \leq \omega^{A}(0)$ then, for all $e \geq 0, \omega^{B}(e) \geq \omega^{A}(e)$.

Proof. By definition of $\omega^{A}($.$) and \tilde{\omega}(),. \omega^{A}(0)>\omega^{B}(0)$ and $f\left(\omega^{A}(0), 0, a\left(\omega^{A}(0), 0\right)\right)=f\left(\omega^{B}(0), 0, b\right)$ entail $a\left(\omega^{A}(0), 0\right)<b=a(\tilde{\omega}(0), 0)$ and thus $\omega^{A}(0)<\tilde{\omega}(0)$. $\tilde{\omega}($.$) is differentiable for all e \geq 0$ and $\tilde{\omega}^{\prime}=-\frac{a_{e}^{\prime}}{a_{w}^{\prime}}<0$ so that $\tilde{\omega}($.$) is strictly decreasing for all e \geq 0$. Since $\omega^{A}($.$) is itself contin-$ uous and strictly increasing for all $e \geq 0$, there exists a unique $\tilde{e}$ satisfying $\tilde{\omega}(\tilde{e})=\omega^{A}(\tilde{e})$. For all $e<\tilde{e}, \tilde{\omega}(e)>\omega^{A}(e) \Rightarrow a(\tilde{\omega}(e), e)>a\left(\omega^{A}(e), e\right)$. Yet, by definition of $\tilde{\omega}(),. a(\tilde{\omega}(e), e)=b$ so that for all $e<\tilde{e}, b>a\left(\omega^{A}(e), e\right)$ and $\omega^{B}(e)<\omega^{A}(e)$ by lemma A3. For all $e \geq \tilde{e}, \tilde{\omega}(e) \leq \omega^{A}(e) \Rightarrow a(\tilde{\omega}(e), e) \leq$ $a\left(\omega^{A}(e), e\right)$. From which it follows that for all $e \geq \tilde{e}, b \leq a\left(\omega^{A}(e), e\right)$ and $\omega^{B}(e) \geq \omega^{A}(e)$. Let us turn to the second part of the lemma. $\omega^{B}(0) \geq \omega^{A}(0)$ 
entails $a\left(\omega^{A}(0), 0\right) \geq b=a(\tilde{\omega}(0), 0)$ and then $\omega^{A}(0) \geq \tilde{\omega}(0)$. Since, for all $e \geq 0, \omega^{A}($.$) is strictly increasing while \tilde{\omega}($.$) is strictly decreasing, it must be$ the case that, for all $e>0, \omega^{A}(e) \geq \tilde{\omega}(e)$. With $a($.$) strictly increasing in$ $w$, for all $e>0, a\left(\omega^{A}(e), e\right) \geq a(\tilde{\omega}(e), e)$. But, by definition of $\tilde{\omega}($.$) , for all$ $e \geq 0, a(\tilde{\omega}(e), e)=b$ so that, for all $e \geq 0, a\left(\omega^{A}(e), e\right) \geq b$ which, by lemma A3, implies $\omega^{A}(e) \leq \omega^{B}(e)$.

Lemma A5 $\frac{d \omega^{A}}{d e}(\tilde{e})<\frac{d \omega^{B}}{d e}(\tilde{e})$.

Proof. The difference is:

$$
\frac{d \omega^{B}}{d e}(\tilde{e})-\frac{d \omega^{A}}{d e}(\tilde{e})=-\frac{\frac{\partial u^{B}}{\partial e}\left(\omega^{B}(\tilde{e}), \tilde{e}\right)}{\frac{\partial u^{B}}{\partial w}\left(\omega^{B}(\tilde{e}), \tilde{e}\right)}+\frac{\frac{\partial u^{A}}{\partial e}\left(\omega^{A}(\tilde{e}), \tilde{e}\right)}{\frac{\partial u^{A}}{\partial w}\left(\omega^{A}(\tilde{e}), \tilde{e}\right)}
$$

For $\omega^{A}(\tilde{e})=\omega^{B}(\tilde{e})=\tilde{\omega}(\tilde{e})=\tilde{w}$, it rewrites:

$\frac{d \omega^{B}}{d e}(\tilde{e})-\frac{d \omega^{A}}{d e}(\tilde{e})=-\frac{\frac{\partial u^{B}}{\partial e}(\tilde{w}, \tilde{e})}{\frac{\partial u^{B}}{\partial w}(\tilde{w}, \tilde{e})}+\frac{\frac{\partial u^{A}}{\partial e}(\tilde{w}, \tilde{e})}{\frac{\partial u^{A}}{\partial w}(\tilde{w}, \tilde{e})}$,

$\frac{d \omega^{B}}{d e}(\tilde{e})-\frac{d \omega^{A}}{d e}(\tilde{e})=-\frac{f_{e}^{\prime}(\tilde{w}, \tilde{e}, b)}{f_{w}^{\prime}(\tilde{w}, \tilde{e}, b)}+\frac{f_{e}^{\prime}(\tilde{w}, \tilde{e}, a(\tilde{w}, \tilde{e}))+f_{a}^{\prime}\left(\tilde{w}, \tilde{e}, a(\tilde{w}, \tilde{e}) a_{e}^{\prime}(\tilde{w}, \tilde{e})\right.}{f_{w}^{\prime}(\tilde{w}, \tilde{e}, a(\tilde{w}, \tilde{e}))+f_{a}^{\prime}(\tilde{w}, \tilde{e}, a(\tilde{w}, \tilde{e})) a_{w}^{\prime}(\tilde{w}, \tilde{e})}$,

and, since $a(\tilde{w}, \tilde{e})=a(\tilde{\omega}(\tilde{e}), \tilde{e})=b$, by definition of $\tilde{\omega}($.$) :$

$$
\frac{d \omega^{B}}{d e}(\tilde{e})-\frac{d \omega^{A}}{d e}(\tilde{e})=-\frac{f_{e}^{\prime}(\tilde{w}, \tilde{e}, b)}{f_{w}^{\prime}(\tilde{w}, \tilde{e}, b)}+\frac{f_{e}^{\prime}(\tilde{w}, \tilde{e}, b)+f_{a}^{\prime}(\tilde{w}, \tilde{e}, b) a_{e}^{\prime}(\tilde{w}, \tilde{e})}{f_{w}^{\prime}(\tilde{w}, \tilde{e}, b)+f_{a}^{\prime}(\tilde{w}, \tilde{e}, b) a_{w}^{\prime}(\tilde{w}, \tilde{e})} .
$$

Neglecting functions' arguments, with obvious writings, one obtains:

$$
\frac{d \omega^{B}}{d e}(\tilde{e})-\frac{d \omega^{A}}{d e}(\tilde{e})=\frac{\tilde{f}_{w}^{\prime} \tilde{f}_{a}^{\prime} \tilde{a}_{e}^{\prime}-\tilde{f}_{e}^{\prime} \tilde{f}_{a}^{\prime} \tilde{a}_{w}^{\prime}}{\left(\tilde{f}_{w}^{\prime}+\tilde{f}_{a}^{\prime} \tilde{a}_{w}^{\prime}\right) \tilde{f}_{w}^{\prime}}>0 .
$$

Lemma $\mathbf{A 6}\left(w^{*}, e^{*}\right) \neq(\tilde{w}, \tilde{e})$.

Proof. Let us consider two pairs of marginal deviations: $\left(d_{A} w, d_{A} e\right)$ defined by $\frac{d_{A} w}{d_{A} e}=\frac{d \omega^{A}}{d e}(\tilde{e})$ (that is such that $\left.d u^{A}=0\right)$ and $\left(d_{B} w, d_{B} e\right)$ defined by $\frac{d_{B} w}{d_{B} e}=\frac{d \omega^{B}}{d e}(\tilde{e})$ (that is such that $\left.d u^{B}=0\right)$. Let us assume $\left(w^{*}, e^{*}\right)=(\tilde{w}, \tilde{e})$. if $S^{\prime}(\tilde{e})>\frac{d \omega^{B}}{d e}(\tilde{e})$ then, for $d_{B} w, d_{B} e>0, S^{\prime}(\tilde{e}) d_{B} e-d_{B} w=\left(S^{\prime}(\tilde{e})-\frac{d \omega^{B}}{d e}(\tilde{e})\right)$ $d_{B} e>0$. If $\quad \frac{d \omega^{B}}{d e}(\tilde{e}) \geq S^{\prime}(\tilde{e})>\frac{d \omega^{A}}{d e}(\tilde{e})$ then, for $\quad d_{A} w, d_{A} e>0$, $S^{\prime}(\tilde{e}) d_{A} e-d_{A} w=\left(S^{\prime}(\tilde{e})-\frac{d \omega^{A}}{d e}(\tilde{e})\right) d_{A} e>0 . \quad$ If $\quad \frac{d \omega^{B}}{d e}(\tilde{e})>S^{\prime}(\tilde{e}) \geq \frac{d \omega^{A}}{d e}(\tilde{e})$ then, for $\quad d_{B} w, d_{B} e<0, S^{\prime}(\tilde{e}) d_{B} e-d_{B} w=\left(S^{\prime}(\tilde{e})-\frac{d \omega^{B}}{d e}(\tilde{e})\right) d_{B} e>0 . \quad$ If $\frac{d \omega^{A}}{d e}(\tilde{e})>S^{\prime}(\tilde{e})$ then, for $d_{A} w, d_{A} e<0, S^{\prime}(\tilde{e}) d_{A} e-d_{A} w=\left(S^{\prime}(\tilde{e})-\frac{d \omega^{A}}{d e}(\tilde{e})\right) d_{A} e$ 
$>0$. In every case, one can find a profitable deviation from $\left(w^{*}, e^{*}\right)$, a contradiction. Consequently, $\left(w^{*}, e^{*}\right) \neq(\tilde{w}, \tilde{e})$.

As a consequence, $u($.$) is differentiable in \left(w^{*}, e^{*}\right)$.

\section{Proof of proposition 1}

Proof. $\lim _{e \rightarrow 0} S^{\prime}(e)=+\infty$ excludes a corner solution. The Lagrangian writes $L(w, e ; \lambda)=S(e)-w+\lambda(u(w, e)-\underline{u}), \lambda>0$. By previous lemma, $\left(w^{*}, e^{*}\right) \neq(\tilde{w}, \tilde{e})$ so that $u($.$) is differentiable in \left(w^{*}, e^{*}\right)$. First order conditions, thus, entail $\left(w^{*}, e^{*}\right) \in\left\{\left(w^{A}, e^{A}\right),\left(w^{B}, e^{B}\right)\right\}$. We just have to check that our assumptions guarantee that first order optimality conditions be sufficient optimality conditions. The second order condition writes:

$$
d^{2} L\left(w^{*}, e^{*} ; \lambda\right)=\lambda(\underbrace{u_{w^{2}}^{\prime \prime}\left(w^{*}, e^{*}\right)}_{\leq 0} d w^{2}+2 \underbrace{u_{w e}^{\prime \prime}\left(w^{*}, e^{*}\right)}_{\leq 0} d w d e+\underbrace{u_{e^{2}}^{\prime \prime}\left(w^{*}, e^{*}\right)}_{\leq 0} d e^{2})+S_{\leq 0}^{\prime \prime}\left(e^{*}\right) d e^{2}
$$

and so the conclusion.

\section{Proof of proposition 2}

Proof. $\omega^{A}(0) \leq \omega^{B}(0) \Leftrightarrow \underline{u} \geq u(\tilde{\omega}(0), 0)$. By lemma A4, $\underline{u} \geq u(\tilde{\omega}(0), 0) \Rightarrow$ $\forall e>0, \omega^{B}(e)>\omega^{A}(e)$ so that $\omega^{B}\left(e^{B}\right)>\omega^{A}\left(e^{B}\right)$. Consequently, $S\left(e^{B}\right)-$ $\omega^{B}\left(e^{B}\right)<S\left(e^{B}\right)-\omega^{A}\left(e^{B}\right)$. But by definition of $e^{A}, \quad S\left(e^{B}\right)-\omega^{A}\left(e^{B}\right) \leq$ $S\left(e^{A}\right)-\omega^{A}\left(e^{A}\right)=\pi^{A}$. Hence, $\pi^{A}>\pi^{B}$ and $\left(w^{*}, e^{*}\right)=\left(w^{A}, e^{A}\right) . \underline{u}<u(\tilde{\omega}(0), 0)$ ensures that $\tilde{e}>0 . \quad S^{\prime}(\tilde{e})>\frac{d \omega^{B}}{d e}(\tilde{e}) \Rightarrow e^{B}>\tilde{e}$. By lemma A4, $\forall e>\tilde{e}$, $\omega^{B}(e)>\omega^{A}(e) \quad$ so that $\omega^{B}\left(e^{B}\right)>\omega^{A}\left(e^{B}\right)$. As a result, $S\left(e^{B}\right)-$ $\omega^{B}\left(e^{B}\right)<S\left(e^{B}\right)-\omega^{A}\left(e^{B}\right) \leq S\left(e^{A}\right)-\omega^{A}\left(e^{A}\right)=\pi^{A}$ by definition of $e^{A}$ : $\left(w^{*}, e^{*}\right)=\left(w^{A}, e^{A}\right) . \quad S^{\prime}(\tilde{e})<\frac{d \omega^{A}}{d e}(\tilde{e}) \Rightarrow e^{A}<\tilde{e} . \quad$ By lemma A4, $\forall e>\tilde{e}$, $\omega^{B}(e)<\omega^{A}(e)$ so that $\omega^{B}\left(e^{A}\right)<\omega^{A}\left(e^{A}\right)$. As a result, $S\left(e^{A}\right)-\omega^{A}\left(e^{A}\right)<$ $S\left(e^{A}\right)-\omega^{B}\left(e^{A}\right) \leq S\left(e^{B}\right)-\omega^{B}\left(e^{B}\right)=\pi^{B}$ by definition of $e^{B}:\left(w^{*}, e^{*}\right)=$ $\left(w^{B}, e^{B}\right)$. Function $\omega()=.\min \left\{\omega^{A}(),. \omega^{B}().\right\}$ is strictly increasing. We thus just have to show that $e^{A}>e^{B}$. $\frac{d \omega^{B}}{d e}(\tilde{e})>S^{\prime}(\tilde{e}) \geq \frac{d \omega^{A}}{d e}(\tilde{e}) \Rightarrow e^{B}<\tilde{e} \leq e^{A}$ while $\frac{d \omega^{B}}{d e}(\tilde{e}) \geq S^{\prime}(\tilde{e})>w^{B}=\omega\left(e^{B}\right)<w^{A}=\omega\left(e^{A}\right)$. Hence, in every case, $e^{B}<e^{A}$ and $w^{B}=\omega\left(e^{B}\right)<w^{A}=\omega\left(e^{A}\right)$.

\section{A.2 Jobs productivity and working conditions}

Proof. By definition of $\left(\underline{e}^{A}, \bar{e}^{B}\right)$ and $\tilde{\psi}, \pi^{A}=\pi^{B}$ and the principal is indifferent between $\left(\underline{w}^{A}, \underline{e}^{A}\right)$ and $\left(\bar{w}^{B}, \bar{e}^{B}\right)$. For the sake of clarity, let us remove the identity superscripts so long identity is not an issue (relations considered hold 
whatever $X \in\{A, B\})$. Profit writes $\pi^{*}=S\left(e^{*}\right)-\omega\left(e^{*}\right)$ so that the impact of some (small) variation $\delta \psi$ is: $\delta \pi=\delta S\left(e^{*}\right)-\delta \omega\left(e^{*} ; \psi\right)$ with $\delta S\left(e^{*}\right)=S^{\prime}\left(e^{*}\right) \delta e^{*}$ and $\delta \omega\left(e^{*} ; \psi\right)=\omega^{\prime}\left(e^{*} ; \psi\right) \delta e^{*}+\delta_{e^{*}} \omega\left(e^{*} ; \psi\right)$ where $\delta_{e} \omega(e ; \psi)$ is a variation of $\omega(e ; \psi)$ for e kept unchanged. Thus

$$
\delta \pi^{*}=\left(S^{\prime}\left(e^{*}\right)-\omega^{\prime}\left(e^{*} ; \psi\right)\right) \delta e^{*}-\delta_{e^{*}} \omega\left(e^{*} ; \psi\right) .
$$

And yet, by definition of $e^{*}, S^{\prime}\left(e^{*}\right)=\omega^{\prime}\left(e^{*} ; \psi\right)$ so that $\delta \pi^{*}=-\delta_{e^{*} \omega}\left(e^{*} ; \psi\right)$. By definition of $\omega($.$) ,$

$$
f\left(\omega(e ; \psi), \frac{e}{\psi} ; x(\omega(e ; \psi), e)\right)=\underline{u},
$$

so that, in $e=e^{*}$, with convenient short writings:

$$
\begin{aligned}
f_{w}^{* \prime} \delta_{e^{*} \omega}\left(e^{*} ; \psi\right)-\frac{e^{*}}{\psi^{2}} f_{e}^{* \prime} \delta \psi+f_{x}^{* \prime} x_{w}^{* \prime} \delta_{e^{*} \omega}\left(e^{*} ; \psi\right) & =\delta \underline{u}=0, \\
\delta_{e^{*}} \omega\left(e^{*}\right) & =\underbrace{\frac{f_{e}^{* \prime}}{f_{w}^{* \prime}+f_{x}^{* \prime} x_{w}^{* \prime}} \frac{e^{*}}{\psi^{2}}}_{<0} \delta \psi .
\end{aligned}
$$

Reinserting identity superscripts, and considering the initial situation, we obtain

$$
\begin{aligned}
\delta_{\underline{e}^{A}} \pi^{A} & =\underbrace{-\frac{f_{e}^{A^{\prime}}}{f_{\bar{w}}^{A^{\prime}}+f_{\bar{x}}^{A^{\prime}} a_{\bar{w}}^{\frac{A^{\prime}}{\prime}}} \frac{\underline{e}^{A}}{\psi^{2}}}_{>0} \delta \psi, \\
\delta_{\bar{e}^{B}} \pi^{B} & =\underbrace{-\frac{f_{e}^{\bar{B} \prime}}{f_{w}^{\bar{B} \prime}} \frac{\bar{e}^{B}}{\psi^{2}}}_{>0} \delta \psi .
\end{aligned}
$$

Consider a variation $\delta \psi>0$. This leads the principal to choose the contract inducing a non pecuniary work motivation if and only if

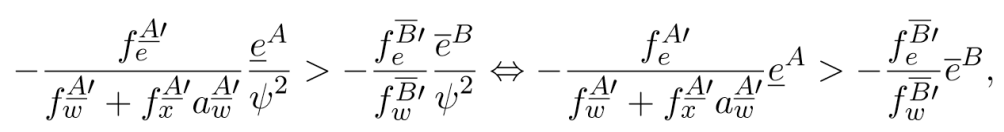

that is, since $S^{\prime}\left(e^{A}\right)=\frac{d \omega^{A}}{d e}\left(e^{A}\right)=-\frac{f_{e}^{A \prime}+f_{x}^{A \prime} a_{e}^{\prime}}{f_{w}^{A \prime}+f_{x}^{A \prime} a_{w}^{\prime}}$, and $S^{\prime}\left(e^{B}\right)=\frac{d \omega^{B}}{d e}\left(e^{B}\right)=-\frac{f_{e}^{B \prime}}{f_{w}^{B \prime}}$ :

so the conclusion.

$$
\frac{S^{\prime}\left(\underline{e}^{A}\right) \underline{e}^{A}}{S^{\prime}\left(\bar{e}^{B}\right) \bar{e}^{B}}=\frac{1}{\tilde{\rho}}>1-\left|\frac{f_{\tilde{x}}^{A^{\prime}} a_{e}^{A_{e}^{\prime}}}{f_{\bar{e}}^{A^{\prime}}}\right|
$$




\section{A.3 Male-female disparities in the labor market: the role of jobs productivity}

We denote $J^{A}$ the set of jobs of type $A$ and $n^{A}$ its cardinality. The fact that all male workers be employed involves $n^{A} \geq n$ and then $\gamma_{n^{B}+1} \geq \tilde{\gamma}$. Let $J_{n}$ be a subset of $n$ jobs. There are $\frac{n^{A} !}{n !\left(n^{A}-n^{11}\right.}$ distinct subsets of cardinality $n$ in $J^{A}$; let these subsets be indexed by $k=1, \ldots, K=\frac{n^{A} !}{n !\left(n^{A}-n\right) !}$. We define $J_{n^{A}-n}(k)=J^{A}-J_{n}(k)$ i.e. for all $k, J_{n}(k)$ and $J_{n^{A}-n}(k)$ form a partition of $J^{A}$. For any subset $J$ of the set of jobs, $\bar{\gamma}(J) \in R_{+}^{*}$ gives the average productivity of jobs in $J$.

Proof. Let $\bar{\gamma}_{1}^{A}$ denote an ex post average productivity of jobs held by male workers.

$$
E \bar{\gamma}_{1}^{A}=\frac{1}{K} \sum_{k=1}^{K} \bar{\gamma}\left(J_{n}(k)\right)=\frac{1}{K} \sum_{k=1}^{K}\left(\frac{1}{n} \sum_{j \in J_{n}(k)} \gamma_{j}\right)=\frac{1}{K} \frac{1}{n} \sum_{k=1}^{K} \sum_{j \in J_{n}(k)} \gamma_{j}
$$

The number of subsets $J_{n}$ such that $j \in J_{n}$ is the number of subsets of $J^{A}-\{j\}$ with $n-1$ elements that is $\frac{\left(n^{A}-1\right) !}{(n-1) !\left(\left(n^{A}-1\right)-(n-1)\right) !}$. This holds for any $j \in J^{A}$ so that:

$$
\sum_{k=1}^{K} \sum_{j \in J_{n}(k)} \gamma_{j}=\frac{\left(n^{A}-1\right) !}{(n-1) !\left(\left(n^{A}-1\right)-(n-1)\right) !} \sum_{j \in J^{A}} \gamma_{j}
$$

As a consequence:

$$
\begin{aligned}
E \bar{\gamma}_{1}^{A} & =\frac{n !\left(n^{A}-n\right) !}{n^{A} !} \frac{1}{n} \frac{\left(n^{A}-1\right) !}{(n-1) !\left(\left(n^{A}-1\right)-(n-1)\right) !} \sum_{j \in J^{A}} \gamma_{j} \\
E \bar{\gamma}_{1}^{A} & =\frac{1}{n^{A}} \sum_{j \in J^{A}} \gamma_{j}=\bar{\gamma}^{A} .
\end{aligned}
$$

By a similar reasoning, we show that $E \bar{\gamma}_{0}^{A}=\frac{1}{K} \sum_{k=1}^{K} \bar{\gamma}\left(J_{n^{A}-n}(k)\right)=\bar{\gamma}^{A}$. The last step is to observe that $E \bar{\gamma}_{1}=E \bar{\gamma}_{1}^{A}=\bar{\gamma}^{A}$ whereas $E \bar{\gamma}_{0}=\frac{n_{B}}{n} \bar{\gamma}_{0}^{B}+\frac{n-n_{B}}{n} E \bar{\gamma}_{0}^{A}$ with $n_{B} \leq n$ and $\bar{\gamma}_{0}^{B}<E \bar{\gamma}_{0}^{A}=\bar{\gamma}^{A}$. Hence, $E \bar{\gamma}_{1}>E \bar{\gamma}_{0}$. Furthermore, the gap is increasing in $n_{B}$. 